\title{
Correction to: A Geometric Algebra Based Higher Dimensional Approximation Method for Statics and Kinematics of Robotic Ma- nipulators
}

Sudharsan Thiruvengadam* and Karol Miller

Correction to: Adv. Appl. Clifford Algebras (2020) 30:17 https://doi.org/10.1007/s00006-019-1039-z

Section 3.1.1. Typographic error in Eq. (41c): the subscript ' 3 ' in ' $\delta_{3}$ ' (coloured in red) should be a ' 4 ' to give ' $\delta_{4}$ '

Section 3.2.2. Typographic error in Eqs. (45c-45h): there is a double wedge ' $\bigwedge \wedge$ ' symbol in the centre of each equation which should be replaced with a single wedge ' $\bigwedge$ ' symbol.

Publisher's Note Springer Nature remains neutral with regard to jurisdictional claims in published maps and institutional affiliations.

Sudharsan Thiruvengadam and Karol Miller

The University of Western Australia, BlueStem Pty. Ltd.

35 Stirling Highway, Crawley

Perth WA 6009

Australia

e-mail: dan.thiru@bluestem.com.au

Sudharsan Thiruvengadam

BlueStem Pty. Ltd.

128 Parry Avenue, Bull Creek

Perth WA 6149

Australia

The original article can be found online at https://doi.org/10.1007/s00006-019-1039-z.

${ }^{*}$ Corresponding author. 